\title{
Comparison of uterine and ovarian stromal blood flow in patients with polycystic ovarian syndrome
}

\author{
Ladan Younesi', Zeinab Safarpour Lima' ${ }^{1}$, Azadeh Akbari Sene², Zahra Hosseini Jebelli' and Ghazaleh Amjad ${ }^{1}$ \\ ${ }^{1}$ Shahid Akbarabadi Clinical Research Development Unit (ShACRDU), Iran University of Medical Sciences (IUMS), Tehran, Iran \\ 2Department of Obstetrics and Gynecology, IVF Fellowship, Shahid Akbar-Abadi Hospital IVF Center, Iran University of Medical Sciences, Tehran, Iran \\ Correspondence should be addressed to Z Safarpour Lima: safarpourlima.z@iums.ac.ir
}

\begin{abstract}
Polycystic ovarian syndrome (PCOS) is one of the most common endocrine disorders. The aim of this study was to find the correlation between color Doppler ultrasound and serum tests as auxiliary diagnostic criteria in areas where there is no possibility of some tests. A total of 108 patients were enrolled. They were divided into three groups including patients with PCOS, patients with PCOA ultrasound, patients with ovaries and normal hormone tests. Transvaginal sonography was performed from three groups and the results were evaluated in gray scale. The volume of the ovary, the number of follicles and the placement of follicles were recorded using using Doppler spectrum of uterine artery and ovarian stroma. Their arterial resistance index was also calculated. In the next step, serum samples were evaluated to determine the level of LH, FSH, free testosterone, DHEAS and 17-OHP hormones in the early follicular phase. Gray scale ultrasonographic findings (volume and number of ovarian follicles) as well as LH values were higher in patients with PCOS than those in the other two groups. These results proved the reliability of using these factors in the prediction of PCOS. In this study, Doppler indexes did not correlate with the size of the ovaries, the number of ovarian follicles and the measured hormone levels. The findings of transvaginal ultrasound and investigating the relationship with clinical and laboratory outcomes, a more suitable pattern could be chosen for more accurate patient selection and, leading to timely treatment and reducing the complications of the disease.
\end{abstract}

\author{
Key Words \\ - polycystic ovary syndrome \\ - transvaginal sonography \\ - color Doppler \\ ultrasonography \\ - resistance index \\ - ovarian
}

\section{Introduction}

Polycystic ovarian syndrome (PCOS) is one of the most common endocrinopathies in humans, which affects five to ten percent of women in childbearing age (1). About 20 to 30 percent of women in reproductive age show polycystic ovaries in ultrasound (2). The three main causes of these patients' referral are infertility (74\%), irregular menstrual cycles (66\%) and an increase in the level of androgens (48\%) (3). It has been defined that infertility was one of the main complaints related to PCOS (4).
Much epidemiologic evidence suggests that PCOS is the most common cause of ovarian dysfunction (POI), which is associated with an increased risk of infertility (5).

Insulin resistance and obesity are also considered to be PCOS disorders and independently increase the risk of abortion and reduce the probability of pregnancy and the birth of a live fetus (6). The risk of hyperplasia and endometrial cancer is higher in women with PCOS. This is due to the unopposed estrogen along with ovulation
This work is licensed under a Creative Commons Attribution-NonCommercial-NoDerivatives 4.0 elnternationab ticense.ifica.com at 04/26/2023 11:35:45AM 
cycles, the lack of progesterone inhibition on endometrial proliferation and the absence of differentiation into the secretion phase (7). Of course, PCOS should no longer be considered just as a gynecologic condition, because many of these women are still at risk of developing cardiovascular disease (8), due to many factors such as obesity, insulin resistance, high blood pressure and fat (9), where a 7.4-fold increase in their mortality was estimated (10).

In multiple analyses the risk of pregnancy, preeclampsia and gestational diabetes mellitus in PCOS women is at least three times higher (11). The risk of early delivery in these individuals is at least double, and their infants have had twice the risk of NICU admission; moreover, their mortality rate is also tripled (12). In addition to metabolic and cardiovascular disorders, psychological disorders and a decrease in the quality of life can be observed in PCOS patients, compared to healthy women, where they are more at risk for depression and anxiety (13). Determination of diagnostic criteria for PCOS plays a key role in early diagnosis and treatment of this condition.

Since the polycystic ovary is an important component of the clinical manifestation, it should be noted that the intra-observer variability and inter have been reported in ultrasound (15). Therefore, the addition of more diagnostic cryotherapy to ultrasound assessment can be very helpful in diagnostic and therapeutic decision making. The total number of follicles and their distribution in gray scale ultrasound are two examples. On the other hand, it has been shown that significant changes have been occurred in vascularization of the ovary and in the surface of the ovarian arteries in patients with PCOS. On the other hand, finding the association between increased androgens and biochemical tests with PCOS ultrasound criteria can be a confirmation stamp for a more accurate diagnosis, as well as a better fit for follow-up, treatment and reducing the number of suspected cases (16).

Consideration should be given to some issues such as the increasing incidence of PCOS, the lack of diagnostic criteria in this issue, the controversial nature of the diagnosis of this syndrome or the high cost of serological tests. If these Doppler ultrasonographic criteria have a meaningful relationship with serum hormone tests, then these criteria could be considered as an auxiliary diagnostic criterion in areas where these tests are not available. Therefore, in this study, we tried to explore the ultrasound criteria of this syndrome in comparison with those who did not have any criteria and to improve the diagnostic criteria of this syndrome, where can be easily assessed by ultrasound, providing an inexpensive and affordable diagnosis.

\section{Materials and methods}

\section{Ethical aspects}

The study was conducted according to the guidelines laid down in the Declaration of Helsinki, and all procedures were approved by the Iran University of Medical Science Ethics Committee and the. Written informed consent was obtained from all patients.

This case-control study was performed using convenience sampling method. The population for entering the research was those who referred to the department of obstetrics and gynecology, Akbar-Abadi Hospital, Tehran between 2013 and 2014 for various reasons such as infertility and having ultrasound criteria for PCOS.

\section{Exclusion criteria}

A) Individuals with systemic conditions include: (1) hypertension above 140/90 $\mathrm{mmHg}$; (2) increased blood lipids; (3) hyperprolactinemia.

B) People with metabolic diseases included (1) diabetes; (2) thyroid problems; (3) adrenals; (4) insulin resistance; (5) acanthose HAIRAN syndrome: hyperandrogenic insulin-resistant acanthosis nigricans.

C) Consumers of anti-lepitic drugs such as: (1) Valproic acid; (2) high-dose exogenous androgens; (3) oral contraceptive pills.

D) Individuals with androgen-secreting tumors.

E) Subjects with congenital adrenal hyperplasia.

F) People with Cushing's syndrome and people who have undergone hormonal treatments during the past 6 months.

The reasons for the exclusion of subjects from the plan was recorded as clear as possible to determine the impact of them and the probable degree of bias and to determine their impact on the results by performing sensitivity analysis. Overall, 108 subjects entered the study. Written consents were taken from people who had eligibility criteria and a complete history was obtained to assess the level of TSH, free T4, triglyceride, cholesterol and so forth. Moreover, ultrasound was performed by Siemens Sonoline G50 Ultrasound with Probe, and ultrasonographic, demographic laboratory data were recorded in the
This work is licensed under a Creative Commons Attribution-NonCommercial-NoDerivatives 4.0 Internationab sicense.ifica . com at $04 / 26 / 2023$ 11:35:45AM 
questionnaire. Transvaginal ultrasonography (TVU) was performed in early follicular phase (between two and five cycles), and then the patients were tested for serum levels of luteinizing hormones, FSH, prolactin (PRL), estradiol, free testosterone, $17 \alpha$-hydroxyprogesterone (17-OHP) and DHEAS.

To reduce the effects of external factors on blood flow, all patients had a 15 -min rest in the waiting room before starting the Doppler and completely empty their bladder. The Ferriman-Gallwey scale hirsutism was measured for the participants of the study as modified by Hatch et al. (17), and the subjects were finally divided into three groups:

Group 1: Patients with PCOS according to Rotterdam diagnostic criteria, where clinical and hormonal condition were normal (PCOS: 45 subjects).

People who exhibited a sonographic picture of the polycystic ovary, while were clinically and hormonally normal (PCO appearance: 31 subjects).

Group 3: Individuals who had normal sonography of the ovaries with normal clinical condition and normal hormonal profiles (32 subjects).

Patient's history, clinical examinations and demographic factors, menstruation, hirsutism were recorded in the questionnaire. A number of variable were investigated in the gray scale including the involved ovarian variables, ovarian volume, number of follicles and placement of follicles. Furthermore, the volume of both ovaries was also calculated using the following formula: thickness $\times$ width $\times$ length $\times 0.523$. The number of follicles was counted in both the anterior and posterior sections of the ovary, and the colon Doppler revealed uterine artery in the cervico-corporial junction and the resistance index (RI) of the uterine artery was examined and recorded separately. By using low pulse repetition frequency setting, a low wall filter and optimum gain, ovarian stromal vascularity and RI were obtained.

\section{Statistical analysis}

In order to evaluate the qualitative variables, frequency was used and quantitative variables such as arterial RI, age and mean were presented by standard deviation. Comparison of RIs in PCOS, PCOA and normal group were performed using $t$-test and Mann-Whitney $U$ depending on the existence or non-existence of parametric statistical tests. Comparison of these arterial indexes with other quantitative variables, such as age was conducted using partial correlation tests in order to eliminate the effect of confounding factors on the two variables. In order to indicate the severity of this relationship, correlation coefficients such as Pearson, Kendall or other coefficients were applied based on the requirements and conditions of the variables. Linear regression (stepwise model) and logistic regression (Forward-Wald) model were used to predict independent factors affecting the resistance of the arteries. The ROC curve was also plotted to determine the best cutting point based on the criteria obtained for PCOS diagnosis.

\section{Results}

A total of 108 people were enrolled in the study, of which 45 were randomized based on the Rotterdam criteria in the PCOS group, followed by 31 patients for the PCOA group (PCO appearance) and 32 subjects for the normal group (clinically and sonographically normal). The mean age for subjects in the PCOS group was 28.1 years, followed by 31 years for the PCOA group and 32 for the normal group. The mean BMI was calculated in PCOS (28.1 \pm 4.6$)$, PCOA $(25.7 \pm 3.7)$ and normal groups $(24.3 \pm 3.8) \mathrm{in} \mathrm{kg} / \mathrm{m}^{2}$. BMI in the PCOS group was higher than the other two groups, where showed a significant difference $(P \leq 0.01)$. Regarding menstruation status, in the group of PCOS, out of 45 subjects $5(11.2 \%)$ had regular menstrual periods and 40 others (88.8\%) had menstrual disorder.

Among all subjects enrolled in group PCOA, of 31 patients, 29 (93.8\%) have a regular menstrual periods, and only 2 patients $(6.5 \%)$ showed menstrual disorder. Of the 32 normal subjects, 30 (93.8\%) had regular menstrual periods, and only $2(6.2 \%)$ had amenorrhea.

The prevalence of menstrual disorders in the PCOS group was significantly different in two groups $(P<0.01)$. In the physical examination, $86.6 \%$ of the PCOS group showed mild-to-moderate hirsutism and $41.9 \%$ had a mild hirsutism in the PCOA group, while only $15.6 \%$ of the normal group revealed mild hirsutism, which statistically showed a significant difference between the three groups $(P \leq 0.01)$.

As indicated in Table 1, the mean of ovarian volume in the PCOS group was higher than the PCOA group and the normal group, while the PCOA group showed more ovarian volume as compared to the normal group $(P<0.01)$. In the sonographic survey, gray scale findings revealed that 42 subjects (93.3\%) in the PCOS group and $26(83.9 \%)$ in the PCOA group exhibited polycystic view in both ovaries, among the remaining individuals of both groups, only one polycystic ovary was seen.

This work is licensed under a Creative Commons Attribution-NonCommercial-NoDerivatives 4.0 Internationab ticense.ifica com at $04 / 26 / 2023$ 11:35:45AM 
Table 1 Average ovarian volume in each group.

\begin{tabular}{|c|c|c|}
\hline Groups & Number of subjects & Mean volume of right ovary (cc) \\
\hline PCOS & 45 & $15.5 \pm 6.1$ \\
\hline PCOA & 31 & $12 \pm 4.8$ \\
\hline Normal & 32 & $8.1 \pm 3.2$ \\
\hline
\end{tabular}

As shown in Table 2, there was no statistical difference in the distribution of follicles in the ovaries between the PCOS group and the PCOA group, although a significant difference was found between these groups and normal groups $(P<0.05)$. The mean number of follicles in the ovaries was recorded in the groups of PCOS (18.3 \pm 6.4$)$, PCOA (14.6 \pm 3.7$)$ and normal (7.1 \pm 2.3$)$. The mean number of ovarian follicles in the PCOS group was significantly higher compared with the PCOA group $(P \leq 0.05)$. In addition, this mean in PCOS and PCOA groups was statistically different from the normal group $(P \leq 0.001)$. It is worth noting that by Doppler ultrasound examination of uterine artery and ovarian stroma, the following results were obtained, where there was no statistically significant difference between the groups ( $P$ value $\geq 0.05$ ).

There was no statistically significant difference in the blood flow RI of ovarian stroma $(P \geq 0.05)$. The statistical evaluation of the ultrasound findings revealed that in the PCOS group, the size of the affected ovaries was directly related to the number of ovarian follicles; in other words, with increasing ovarian volume, the number of ovarian follicles also increased $(P \leq 0.001)$. The PCOA group also showed a similar result. In both PCOS PCOA groups, the volume and number of ovarian follicles were not significantly associated with RI of ovarian stroma and RI of the uterine artery. In PCOS and PCOA groups, BMI and age did not correlate with ultrasound data (ovarian volume, number of follicles, RI of the uterine artery and ovarian stromal RI) (Tables 3 and 4).

Based on data presented herein, LH level increased in the majority of PCOS group than PCOA group $(P \leq 0.001)$. In other hormonal studies, no significant difference was found between the two groups $(P \geq 0.05)$. In the PCOS group, no relationship was found between the mean RI of the uterine artery and the RI of the ovarian stroma $(P \geq 0.05)$ (Table 5).

\begin{tabular}{ccc}
\hline Mean volume of left ovary $(\mathrm{cc})$ & & Mean volume of both ovaries $(\mathrm{cc})$ \\
\cline { 1 - 2 } & $16 \pm 5.1$ \\
$12.8 \pm 4.8$ & $12.4 \pm 4.1$ \\
$8.2 \pm 5$ & $8.1 \pm 3.6$ \\
\hline
\end{tabular}

Among the individuals in the PCOS group, no association was found between the levels of hormonal factors (LH, free testosterone, DHEAS and 17-OH progesterone) with an average RI of the ovarian stroma $(P \geq 0.05)$. In the PCOS group, there was no relationship between the mean RI of the uterine artery and the hormonal factors. In the PCOS group, there was no correlation between ovarian volume and hormonal factors, and no correlation was found between the number of ovarian follicles and hormonal factors (Table 5).

In the PCOA group, there was a correlation between LH and ovarian volume $(P=0.03)$. LH levels have increased in individuals with more ovarian volume. The group also demonstrated that the levels of free testosterone, 17-OH progesterone and DHEAS hormones were also related to ovarian volume $(P=0.001)$. Moreover, the levels of free testosterone, 17-OH progesterone and DHEAS were related to ovarian follicles in the PCOA group $(P=0.001)$. The findings of this group suggested that there was no relationship between hormonal factors with ovarian Doppler index and uterine artery $(P \geq 0.05)$. Overall, the mean ovarian volume was significantly correlated with $\mathrm{LH}$, free testosterone, $17-\mathrm{OH}$ progesterone and DHEAS in both PCOS and PCOA groups $(P=0.03)$. The mean number of follicles was linked to the level of free testosterone, $17-\mathrm{OH}$ progesterone and DHEAS $(P$ value $=0.01)$. However, Doppler RI in these two groups did not correlate with hormonal factors $(P \geq 0.05)$ (Table 5$)$.

\section{Discussion}

In this study, we have found that the findings of transvaginal ultrasound together with clinical and laboratory outcomes, a more suitable pattern could be chosen for more accurate patient selection and, leading to

Table 2 Distribution of follicles in the ovaries among the three groups.

\begin{tabular}{lcc}
\hline Groups & Number of subjects \\
\cline { 1 - 1 } PCOS & 45 \\
PCOA & 31 \\
Normal & 32 \\
\hline
\end{tabular}

\begin{tabular}{c}
\hline Number of peripherally located follicles \\
\hline 37 \\
25 \\
15
\end{tabular}

\begin{tabular}{c} 
Number of randomly distributed follicles \\
\hline 8 \\
6 \\
17
\end{tabular}


Table 3 Average RI of the uterine arteries in each group.

\begin{tabular}{lc}
\hline & RI (right uterine artery) \\
\hline PCOS & $0.96 \pm 0.46$ \\
PCOA & $0.87 \pm 0.89$ \\
Normal & $0.87 \pm 0.13$ \\
\hline
\end{tabular}

timely treatment and reducing the complications of the disease.

PCOS is a heterozygote pathological condition, prominent manifestations of which are reproductive disorders, and is commonly associated with hyperandrogenism, obesity, hyperinsulinemia and insulin resistance (18). Ovarian morphologic examination with ultrasound is essential for PCOS diagnosis. A Doppler ultrasound is an ultrasound that measures the amount of blood flow in the vessels and tissues, and criteria for the uterine and ovarian arteries can be measured (19). In this study, a total of 108 had inclusion criteria, which were classified into three categories including PCOS group (45 individuals), PCOA group (31 subjects) and normal group (32 subjects). The mean age for subjects in the PCOS group was 28.1 years, followed by 31 years for the PCOA group and 32 for the normal group. The mean BMI was calculated in PCOS $(28.1 \pm 4.6)$, PCOA $(25.7 \pm 3.7)$ and normal groups $(24.3 \pm 3.8)$ in $\mathrm{kg} / \mathrm{m}^{2}$. BMI in the PCOS group was found to be significantly higher compared with the other two groups $(P \leq 0.01)$. A study by Lam et al. also reported a significant difference in BMI between the PCOS and normal group (20). Regarding the prevalence of menstrual disorders, the prevalence of amenorrhea in the PCOS group $(88.8 \%)$ was significantly higher than that of the PCOA (6.5\%) and normal (6.2\%) groups. Therefore, anovulation and cyclic disorders was the most frequently detected types in the PCOS group, which was expected due to the choice of patients. These results were also consistent with the two Lam and Bostanci studies. The prevalence of menstrual disorders in the Caucasian PCOS group was reported to be 75 and $100 \%$, respectively $(16,20)$. In terms of clinical evidence of hyperandrogenism, the study of hirsutism was performed in a clinical examination and subjects were classified according to the Ferriman-Gallwey Score in three degrees of mild, moderate and severe hirsutism. Based on the data presented herein, $86.6 \%$ of the PCOS group showed mild-to-moderate hirsutism and $41.9 \%$ had a mild hirsutism in the PCOA group, while only $15.6 \%$ of the normal group revealed mild hirsutism, which statistically revealed a significant difference between the three groups $(P \leq 0.01)$. Therefore, hirsutism as criteria for clinical hyperandrogenism was clearly more prevalent in the PCOS group. Our research results were consistent with many studies. Fulghesu in Italy reported an increased rate of hirsutism in 418 patients with PCOS (48.9\%), (21). There was no significant difference between the sonographic findings of bilateral ovaries in the present study. Therefore, the mean sonographic data of ovaries was evaluated and compared between the three groups. According to Rotterdam's ultrasound index, $10 \mathrm{cc}$ is considered as a cut-off point for PCOS. The mean ovarian volume in the PCOS group was $16 \mathrm{cc}$ compared to $12.4 \mathrm{cc}$ in the PCOA group and $8.1 \mathrm{cc}$ in the normal group. Accordingly, the ovaries in the group with PCOS had a higher mean volume than the other two groups. The PCOA group also had a higher volume of ovaries than the normal group. The findings of this study also revealed that the mean volumes in the two groups with polycystic view (PCOA and PCOS) were much higher, where Fulghesu's study also confirmed this evidence (21).

The next factor was the number of follicles smaller than $9 \mathrm{~mm}$ in the ovary, and the mean values obtained in the PCOS group (18.3) were significantly higher than the two groups of PCOA (14.6) and the normal group (7.1). The difference in the number of follicles was statistically significant between the two groups of PCOA and the normal. Lam et al. also reported that the average number of follicles was 15 in the PCOS group and 5.5 in the normal group of Chinese women. They also reported that Caucasian women had an average of 16.3 follicles in the PCOS group vs 5.5 in the normal group, which is consistent with our findings $(20,22)$. The ovarian stroma and uterine artery of all patients were examined

Table 4 Average RI of the ovarian stroma in each group.

\begin{tabular}{lc} 
& $\mathbf{R I}$ (right ovarian stroma) \\
\cline { 1 - 1 } PCOS & $0.49 \pm 0.08$ \\
PCOA & $0.51 \pm 0.06$ \\
Normal & $0.48 \pm 0.09$
\end{tabular}

\begin{tabular}{c} 
RI (left ovarian stroma) \\
\hline $0.51 \pm 0.09$ \\
$0.49 \pm 0.06$ \\
$0.51 \pm 0.08$
\end{tabular}

RI (mean of right and left ovarian stroma)

$0.50 \pm 0.07$

$0.50 \pm 0.05$

$0.50 \pm 0.04$ 
Table 5 Analysis of laboratory data and hormonal findings in PCOS and PCOA groups.

\begin{tabular}{|c|c|c|c|c|}
\hline & \multicolumn{2}{|c|}{ PCOS group $(n=45)$} & \multicolumn{2}{|c|}{ PCOA group $(n=32)$} \\
\hline & Normal & Increase & Normal & Increase \\
\hline $\mathrm{LH}$ & 22 & 23 & 28 & 4 \\
\hline Free testosterone & 37 & 8 & 27 & 5 \\
\hline $\mathrm{OH}-17$ & 36 & 8 & 30 & 2 \\
\hline $\begin{array}{l}\text { Progesterone } \\
\text { DHEAS }\end{array}$ & 37 & 8 & 29 & 3 \\
\hline FSH & 43 & 2 & 32 & 0 \\
\hline
\end{tabular}

by $2 \mathrm{D}$ color Doppler and wave spectra were consequently recorded.

The arterial RI derived from the spectrum of ovarian stroma and uterine arteries on each side were calculated separately. The findings of the two sides showed no significant difference in the statistical scale. Therefore, the average RI of ovarian stroma and RI of the uterine arteries on both sides were statistically analyzed. In the statistical analysis, there was a significant difference between the Doppler indexes in these three groups, which is consistent with the study of Lakhani et al. (3).

The volume of the ovary and the number of follicles were associated with amenorrhea and the incidence of hirsutism; furthermore, higher ovarian volume and higher follicle count were found to markedly increase menstrual disorders and hirsutism, which this pathophysiological finding revealed ovarian morphology with clinical manifestations of hyperandrogenism. However, in this study, RI of uterine artery and ovarian stroma were not correlated with menstrual disorder and hirsutism. These findings were similar to those of Lam et al., (20) and (22), that there has been no correlation between ovarian volume, follicle count, RI of uterine and ovarian arteries with anovulation and hirsutism in PCOS patients $(20,22)$.

Fulghesu et al. evaluated ultrasound findings with hormonal values and they reported that ovarian volume was associated with LH levels in people with PCOS, which showed similar results to the current study outcomes. On the other hand, there was a positive correlation between the numbers of follicles with 17-OH-progesterone in aforementioned study, which was revealed in the present study (21).

In conclusion, the present study was conducted to evaluate the levels of LH, FSH and androgenic hormones free testosterone, 17-OH-progesterone and DHEAS. The percentage of LH above normal in the PCOS group was significantly higher than the PCOA group (48.9 vs $12.5 \%$ ). This difference was not significant in other hormones.

Overall, the mean ovarian volume of both PCOS and PCOA groups was positively associated with abnormal levels of $\mathrm{LH}$, free testosterone, progesterone $17-\mathrm{OH}$ and DHEAS. In addition, the number of follicles was positively associated with abnormal amounts of free testosterone, progesterone-OH-17 and DHEAS. Meanwhile, there was no association between Doppler indexes and hormonal factors.

\section{Declaration of interest}

The authors declare that there is no conflict of interest that could be perceived as prejudicing the impartiality of the research reported.

\section{Funding}

This research did not receive any specific grant from any funding agency in the public, commercial or not-for-profit sector.

\section{Author contribution statement}

$\mathrm{L} Y, \mathrm{Z} \mathrm{S} \mathrm{L}, \mathrm{A} A \mathrm{~S}, \mathrm{Z} \mathrm{H}$ J and $\mathrm{G}$ A planned and designed the study, ran the experiments, ran the analyses, interpreted the results, the manuscript and revised the manuscript; all authors approved the final version of the manuscript.

\section{References}

1 Darvishi M, Forootan M, Azmodeh O, Forootan M \& Matinfar H. Studying the frequency of helicobacter pylori infection among women suffering from polycystic ovary resorting to hospital infertility center. Biomedical and Pharmacology Journal 201710 75-80. (https://doi.org/10.13005/bpj/1083)

2 Pezeshki Rad M, Mohammadifard M, Ravari H, Farrokh D, Ansaripour E \& Saremi E. Comparing color doppler ultrasonography and angiography to assess traumatic arterial injuries of the extremities. Iranian Journal of Radiology 201512 e14258. (https://doi. org/10.5812/iranjradiol.14258)

3 Lakhani K, Seifalian AM \& Atiomo WU. Polycystic ovaries. British Journal of Radiology 200275 9-16. (https://doi.org/10.1259/ bjr.75.889.750009)

4 Stein Leventhal ML. Amenorrhea associated with bilateral polycystic ovaries. American Journal of Obstetrics and Gynecology 193529 181-188. (https://doi.org/10.1016/S0002-9378(15)30642-6)

5 Hull MG. Epidemiology of infertility and polycystic ovarian disease: endocrinological and demographic studies. Gynecological Endocrinology 19871 235-245. (https://doi. org/10.3109/09513598709023610)

6 Palomba S, Santagni S, Falbo A \& La Sala GB. Complications and challenges associated with polycystic ovary syndrome: current perspectives. International Journal of Women's Health 20157 745-763. (https://doi.org/10.2147/IJWH)

7 Navaratnarajah R, Pillay OC, Hardiman P. Polycystic ovary syndrome and endometrial cancer. Seminars in Reproductive Medicine 200826 62-71. (https://doi.org/10.1055/s-2007-992926)

8 Conway GS, Agrawal R \& Bettridge DJ. Risk factors for coronary artery disease in lean and obese women with polycystic ovary syndrome. Clinical Endocrinology 199237 119-125. (https://doi. org/10.1111/j.1365-2265.1992.tb02295.x)

9 McKeigue P. Cardiovascular disease and diabetes in women with polycystic ovary syndrome. Baillière's Clinical Endocrinology and Metabolism 199610 311-318. (https://doi.org/10.1016/S0950351X(96)80155-8) 
10 Pierpoint T, McKeigue PM, Isaacs AJ \& Wild SH. Mortality of women with polycystic ovary syndrome at long-term follow-up. Journal of Clinical Epidemiology 199851 581-586. (https://doi.org/10.1016/ S0895-4356(98)00035-3)

11 Qin JZ, Pang LH, Li MJ, Fan XJ, Huang RD \& Chen HY. Obstetric complications in women with polycystic ovary syndrome: a systematic review and meta-analysis. Reproductive Biology and Endocrinology 201326 11-56. (https://doi.org/10.1186/1477-782711-56)

12 Boomsma CM, Eijkemans MJ \& Hughes EG. A meta-analysis of pregnancy outcomes in women with polycystic ovary syndrome. Human Reproduction Update 200612 673-683. (https://doi. org/10.1093/humupd/dml036)

13 Moran LJ, Deeks AA \& Gibson-Helm ME. Psychological parameters in the reproductive phenotypes of polycystic ovary syndrome. Human Reproduction 201227 2082-2088. (https://doi.org/10.1093/humrep/ des114)

14 Lujan ME, Chizen DR, Peppin AK, Dhir A \& Pierson RA. Assessment of ultrasonographic features of polycystic ovaries is associated with modest levels of inter-observer agreement. Journal of Ovarian Research 20092 6. (https://doi.org/10.1186/1757-2215-2-6)

15 Amer SA, Li TC \& Bygrave C. An evaluation of the inter-observer and intra-observer variability of the ultrasound diagnosis of polycystic ovaries. Human Reproduction 200217 1616-1622. (https://doi. org/10.1093/humrep/17.6.1616)

16 Bostanci MS, Sagsoz N \& Noyan V. Comprasion of ovarian stromal and uterin artery blood flow measured by color doppler ultrasonography in polycystic ovary syndrome patients and patients with ultrasonographic evidence of polycystic. Journal of Clinical
Gynecology and Obstetrics 20132 20-26. (https://doi.org/10.4021/ jcgo85w)

17 Hatch R, Rosenfield RL, Kim MH \& Tredway D. Hirsutism: implications, etiology, and management. American Journal of Obstetrics and Gynecology 1981140 815-830. (https://doi. org/10.1016/0002-9378(81)90746-8)

18 Aziz R, Woods KS \& Reyna R. The prevalence and features of polycystic ovary syndrome in an unselected population. Journal of Clinical Endocrinology and Metabolism 200213 251-257. (https://doi. org/10.1210/jc.2003-032046)

19 Zare Mehrjardi M, Darabi M, Bagheri SM, Kamali K \& Bijan B. The role of ultrasound (US) and magnetic resonance imaging (MRI) in penile fracture mapping for modified surgical repair. International Urology and Nephrology 201749 937-945. (https://doi.org/10.1007/ s11255-017-1550-x)

20 Lam P, Raine-Fenning N, Cheung L \& Haines C. Three-dimensional ultrasound features of the polycystic ovary in Chinese women. Ultrasound in Obstetrics and Gynecology 200934 196-200. (https://doi. org/10.1002/uog.6442)

21 Fulghesu AM, Angioni S, Frau E, Belosi C, Apa R \& Mioni R. Ultrasound in polycystic ovary syndrome-the measuring of ovarian stroma and relationship with circulating androgens: results of a multicentric study. Human Reproduction 200722 2501-2508. (https:// doi.org/10.1093/humrep/dem202)

22 Lam Po M, Johnson Ian R \& Raine-Fenning Nick J. Threedimensional ultrasound features of the polycystic ovary and the effect of different phenotypic expressions on hese parameters. Human Reproduction 200722 3116-3123. (https://doi.org/10.1093/ humrep/dem218)

Received in final form 29 November 2018

Accepted 21 December 2018

Accepted Preprint published online 21 December 2018 https://ec.bioscientifica.com https://doi.org/10.1530/EC-18-0423 (c) 2019 The authors Published by Bioscientifica Ltd
This work is licensed under a Creative Commons Attribution-NonCommercial-NoDerivatives 4.0 Internationab sicense.ifica . com at $04 / 26 / 2023$ 11:35:45AM 